\title{
Normal and Lateral Casimir Forces between Deformed Plates
}

\author{
Thorsten Emig \\ Institut für Theoretische Physik, Universität zu Köln, Zülpicher Straße 77, D-50937 Köln, Germany \\ Andreas Hanke \\ Institut für Theoretische Physik, Universität Stuttgart, \\ Pfaffenwaldring 57, D-70550 Stuttgart, Germany \\ Ramin Golestanian \\ Institute for Advanced Studies in Basic Sciences, Zanjan 45195-159, Iran \\ and Institute for Studies in Theoretical Physics and Mathematics, P.O. Box 19395-5531, Tehran, Iran \\ Mehran Kardar \\ Physics Department, Massachusetts Institute of Technology, Cambridge, MA 02139
}

(Dated: November 10, 2018)

\begin{abstract}
The Casimir force between macroscopic bodies depends strongly on their shape and orientation. To study this geometry dependence in the case of two deformed metal plates, we use a path integral quantization of the electromagnetic field which properly treats the many-body nature of the interaction, going beyond the commonly used pairwise summation (PWS) of van der Waals forces. For arbitrary deformations we provide an analytical result for the deformation induced change in Casimir energy, which is exact to second order in the deformation amplitude. For the specific case of sinusoidally corrugated plates, we calculate both the normal and the lateral Casimir forces. The deformation induced change in the Casimir interaction of a flat and a corrugated plate shows an interesting crossover as a function of the ratio of the mean plate distance $H$ to the corrugation length $\lambda$ : For $\lambda \ll H$ we find a slower decay $\sim H^{-4}$, compared to the $H^{-5}$ behavior predicted by PWS which we show to be valid only for $\lambda \gg H$. The amplitude of the lateral force between two corrugated plates which are out of registry is shown to have a maximum at an optimal wavelength of $\lambda \approx 2.5 \mathrm{H}$. With increasing $H / \lambda \gtrsim 0.3$ the PWS approach becomes a progressively worse description of the lateral force due to many-body effects. These results may be of relevance for the design and operation of novel microelectromechanical systems (MEMS) and other nanoscale devices.
\end{abstract}

PACS numbers: 03.70.+k, 11.10.-z, 42.50.Ct, 12.20.-m

\section{INTRODUCTION}

More than five decades ago, Casimir predicted that the ground state energy of photons is alternated in the presence of two parallel perfectly conducting metal plates in such a way as to lead to an observable macroscopic force between them [1]. The attractive force (per plate area $A$ ) has an universal amplitude, an energy scale set by the fundamental constant $\hbar c$, and decays with the distance $H$ between the plates as

$$
\frac{F}{A}=-\frac{\pi^{2}}{240} \frac{\hbar c}{H^{4}} .
$$

This remarkable prediction of quantum electrodynamics has implications in many contexts ranging from surface physics [2], particle physics [3], to cosmology [4]. Because of its fundamental nature, the Casimir effect has motivated extensive theoretical work, especially during the last decade. The pioneering result in Eq. (1) has been generalized to include important effects such as the finite conductivity and surface roughness of the plates, finite temperature, and even moving plates in the dynamic counterpart to the Casimir effect (see Refs. [5, 6, 6, 8, 9, 10, 11] for reviews).

On the experimental front, the early attempts at observing the Casimir force, initiated by Sparnaay in 1958 [12] and later by Van Blokland and Overbeek in 1978 [13], were not conclusive due to large experimental uncertainty. In recent years, however, there have been a number of precision measurements which set the modern stage in this field; starting in 1997 by Lamoreaux 14 who used a torsion pendulum with an electromechanical feedback system to measure the Casimir force between a spherical surface (lens) and a flat plate. Mohideen et al. 15 measured the Casimir force between a sphere mounted on the tip of a flexible cantilever and a flat plate by an atomic force microscope. Chan et al. 16] measured the Casimir force between a sphere and a flat plate in a microelectromechanical system (MEMS) using a micromachined torsional device. All these experiments confirm the Casimir force formula in the range from $100 \mathrm{~nm}$ to several $\mu \mathrm{m}$ to a few per cent accuracy. In order to achieve this high precision, a careful analysis of the corrections due to the finite conductivity of the metal surfaces, roughness, and nonzero temperature, 
is indispensable 11, 17, 18, 19, 20, 21]. The above cited experiments in fact deviate from the flat plate geometry corresponding to Eq. (11) by using a plane-sphere configuration (thus avoiding the experimental difficulty of keeping two flat surfaces sufficiently parallel). The force for a spherical surface with (large) radius $R$ at a distance $H$ of closest surface-to-surface approach from a flat plate can then be calculated from the Casimir potential $\mathcal{E}(H)$ for two flat plates by using the proximity force rule [22], see Eq. (28) below. Recently, however, G. Bressi et al. 23] measured the Casimir force between two parallel flat surfaces directly, confirming Eq. (1) to $15 \%$ accuracy.

The Casimir force in Eq. (11) has analogies to the effective force between particles or plates immersed in a system close to its critical point, which arises due to the modification of thermal fluctuations of the bulk order parameter. This effect was originally predicted in 1978 by Fisher and de Gennes 24] for colloidal particles immersed in a binary liquid mixture near its critical de-mixing point, and observed experimentally for silica spheres immersed in a mixture of water and oil (2,6-lutidine) [25]. Related phenomena occur in liquid crystals [26], microemulsions [27], and for inclusions in fluctuating membranes [28] (see Ref. 10] for a review). In recent years, the critical-point Casimir effect has attracted increasing theoretical 10, 29, 30, 31, 32, 33] and experimental interest [34, 35]. For He $\mathrm{He}^{4}$ wetting films close to the superfluid phase transition, the theoretical predictions [29] for critical-point Casimir forces between parallel surfaces exhibiting Dirichlet boundary conditions have been confirmed quantitatively [35].

Equation (11) for the electromagnetic Casimir force is valid in the ideal limit of perfectly conducting plates. In the more general context of the Lifshitz theory for dielectric bodies [36], this corresponds to an infinite dielectric constant $\varepsilon$ for all frequencies $\omega$. For finite $\varepsilon=\varepsilon(\omega)$, this power law for the force is recovered for large distances $H \gg c / \omega_{0}$, where $\omega_{0}$ is the smallest resonance (absorption) frequency of the dielectric (usually $c / \omega_{0} \approx 10-100 \mathrm{~nm}$ ). In this, so-called retarded, limit, the force is universal in the sense that it only depends on the electrostatic dielectric constant $\varepsilon_{0}=\varepsilon(0)$. The opposite limit of $H \ll c / \omega_{0}$ gives the unretarded van der Waals force $F / A \sim H^{-3}$. The interpretation of the Casimir force in terms of changes in zero point vacuum electromagnetic energy suggests it to be a strong function of geometry; probing the global shape of the boundary that confines the vacuum fluctuations [37. Indeed, whereas the van der Waals force between electrically polarizable particles is always attractive, even the sign of the Casimir force is geometry dependent, and can be repulsive, e.g., for a thin spherical or cubic conducting shell [38, 39, 40, 41]. Repulsive Casimir forces are expected also when magnetic properties of the boundaries are exploited, e.g., by using a perfectly conducting and an infinitely permeable plate [42].

Apart from the importance of these phenomena to basic science, the ongoing refinement of nanofabrication technology in electronics and mechanics also provides new impetus for the understanding of such systems in view of applications in nanotechnology. On length scales of about $100 \mathrm{~nm}$ and below, the Casimir force becomes comparable or even dominant to other forces [43], and thus must be taken into account in the design and operation of nanoscale devices [44, 45]. Indeed, the experiment of Chan et al. [16] demonstrates the possibility for novel actuation schemes in microelectromechanical systems (MEMS) based on the Casimir force. They also show that the Casimir force can be used to control dynamic properties of such systems, e.g., in a nonlinear micromechanical Casimir oscillator [46]. The above mentioned dependence of strength and sign of the Casimir force on geometry and material properties offers the opportunity to manipulate this interaction in a controlled way, e.g., by tailoring the shape of the interacting surfaces. On the other hand, movable elements of nanoscale devices may unwantedly stick together due to the strong attractive Casimir force, impeding their operation [44, 45. This so-called stiction could possibly be prevented by using shapes (e.g., suitable modulations) and materials of movable elements such that the Casimir force between them is repulsive (or at least reduced).

Due to the importance of the Casimir force to basic and applied science, it is highly desirable to demonstrate its strong shape dependence in a set-up that clearly shows its distinction from the usual pairwise additive interactions [47. In a previous Letter [48] we pointed out that a promising route to this end is via modifications of the parallel plate geometry, since measurement of the putative repulsive Casimir interaction for a conducting sphere is experimentally difficult. In searching for nontrivial boundary dependences, Roy and Mohideen 49] examined the force between a sphere and a sinusoidally corrugated plate with amplitude $a \approx 60 \mathrm{~nm}$ and wavelength $\lambda \approx 1.1 \mu \mathrm{m}$. (This geometry was first suggested in Refs. [47].) Over the range of separations $H \approx 0.1-0.9 \mu \mathrm{m}$, the observed force showed clear deviations from the dependence expected on the basis of decomposing the Casimir force to a sum of pairwise contributions (in effect, an average over the variations in separations). This experimental result motivated our calculation of the exact Casimir force in the geometry depicted in Fig. 1, without the assumption of pairwise additivity. Our analytic results [see Eq. (32) and Fig. 2 hold to second order in $a$, and show that for fixed $H$ the corrections due to corrugation strongly depend on $\lambda$. In fact, for $H / \lambda \gg 1$ the correction is by a factor of $H / \lambda$ larger than in the opposite limit of $H / \lambda \ll 1$ where the assumption of pairwise additivity is asymptotically correct. However, the experiments of Ref. [49] are performed in the range of $H / \lambda \approx 0.1-0.8$ where the corrections to pairwise additivity may not be significant enough to account for the observed deviations. In Ref. [51] it has been suggested that these deviations are due to a lateral force that tends to preferentially position the spherical AFM tip on top of local maxima of the modulated surface (leading to a smaller separation and stronger force). Based on our results, we thus propose that the shape dependence of the Casimir force can be probed more clearly by going to modulations of shorter wavelength; a hard 
but achievable goal.

The originally predicted Casimir force between two flat metal plates (or between a flat and a deformed plate) is, for symmetry reasons, oriented normal to the surfaces of the plates. However, if both plates are deformed there is also a lateral Casimir force, as predicted in Refs. [47] and confirmed experimentally [52]. To date, the lateral force between two corrugated plates has been calculated explicitely only within the approximative approach of a pairwise summation of van der Waals forces, see, e.g., [11]. Here we calculate the lateral force exactly to second order in the corrugation amplitude for the geometry shown in Fig. 1 , without referring to a pairwise summation scheme. As for the normal force, our results [cf. Eq. (46) and Fig. 5] show that the lateral force strongly depends on the ratio of the corrugation length $\lambda$ and mean plate separation $H$. We find the pairwise summation to be a valid approximation only for sufficiently small values of $H / \lambda \lesssim 0.3$. However, the experiment of Ref. 52 is performed at a ratio $H / \lambda \approx 0.18$ where we do not expect significant deviations from the pairwise summation approximation.

The outline of the paper is as follows: In the next section we set up the general path integral formulation for the Casimir energy of the electromagnetic gauge field. By separating into transversal electric and magnetic modes, we reduce the problem to two decoupled problems for scalar fields which differ only in their boundary conditions. In each case, we calculate the Casimir energy for general plate deformations perturbatively. In the third section, we give a brief summary of the pairwise summation approach, and the resulting Casimir interaction. Section four gives detailed results on both the normal and the lateral force between sinusoidally corrugated plates. We conclude by discussing the relevance of our results to experiments. Details of the calculations are left to the appendices.

\section{PATH INTEGRAL FORMULATION OF THE CASIMIR ENERGY}

We consider two perfectly conducting deformed plates $S_{\alpha}(\alpha=1,2)$ of mean separation $H$, which are infinitely extended along the plane spanned by $\mathbf{y}_{\|}=\left(y_{1}, y_{2}\right)$. Assuming static and uniaxial deformations without overhangs, their profiles are described by height functions $h_{\alpha}\left(y_{1}\right)$, with $\int d y_{1} h_{\alpha}\left(y_{1}\right)=0$. The Casimir energy at zero temperature corresponds to the difference of the ground state energies of the quantized electromagnetic (EM) field for plates at distance $H$ and at $H \rightarrow \infty$, respectively. To obtain this energy, we employ the path integral quantization method. For general deformations, it is necessary to consider the action

$$
S_{\mathrm{em}}\left\{A_{\mu}\right\}=-\frac{1}{4} \int d^{4} X F_{\mu \nu} F^{\mu \nu}
$$

where $X$ denotes a point of $4 \mathrm{D}$ spacetime, $F_{\mu \nu}=\partial_{\mu} A_{\nu}-\partial_{\nu} A_{\mu}$, and the four potential $A_{\mu}$ is subject to the boundary condition that the tangential components of the electric field vanish on the surfaces. The redundant degrees of freedom due to the gauge invariance of the electromagnetic field can be eliminated by the Faddeev-Popov gauge fixing procedure 47, 53].

However, for the uniaxial deformations under consideration here, we can develop a simpler quantization scheme, by a similar reasoning as used in the context of waveguides with constant cross-sectional shape [48]. In this case, the transverse magnetic (TM) waves and transverse electric (TE) waves (with respect to the translational invariant $y_{2}$ direction) constitute a complete set of modes to describe an arbitrary electromagnetic field between the plates [54]. For TM waves all field components are then uniquely given by a scalar function corresponding to the electric field along the invariant direction,

$$
\Phi_{\mathrm{TM}}\left(t, y_{1}, y_{2}, z\right)=E_{2}\left(t, y_{1}, y_{2}, z\right)
$$

with the Dirichlet boundary condition $\left.\Phi_{\mathrm{TM}}\right|_{S_{\alpha}}=0$ on each surface $S_{\alpha}$. The TE waves are analogously described by the scalar function

$$
\Phi_{\mathrm{TE}}\left(t, y_{1}, y_{2}, z\right)=B_{2}\left(t, y_{1}, y_{2}, z\right)
$$

with the Neumann boundary condition $\left.\partial_{n} \Phi_{\mathrm{TE}}\right|_{S_{\alpha}}=0$, where $\partial_{n}$ is the normal derivative of the surface $S_{\alpha}$ pointing into the space between the two plates. After a Wick rotation to the imaginary time variable $X^{0}=i c t$, both fields $\Phi_{\mathrm{TM}}$ and $\Phi_{\mathrm{TE}}$ can be quantized using the Euclidean action

$$
S_{\mathrm{E}}\{\Phi\}=\frac{1}{2} \int d^{4} X(\nabla \Phi)^{2} .
$$

In the $4 \mathrm{D}$ Euclidean space, the plates are parameterized by $X_{1}(\mathbf{y})=\left[\mathbf{y}, h_{1}\left(y_{1}\right)\right]$ and $X_{2}(\mathbf{y})=\left[\mathbf{y}, H+h_{2}\left(y_{1}\right)\right]$, where $\mathbf{y}=\left(y_{0}, y_{1}, y_{2}\right)=\left(y_{0}, \mathbf{y}_{\|}\right)$, and $y_{0}=i c t$. 
In order to obtain the ground state energy from this quantization scheme, we now consider the partition functions $\mathcal{Z}_{\mathrm{D}}$ and $\mathcal{Z}_{\mathrm{N}}$ for the scalar field Euclidean action $S_{\mathrm{E}}$ both with Dirichlet (D) and Neumann (N) boundary conditions at the surfaces. Following Refs. [47, 55] (cf. also Ref. [56]), we implement the boundary conditions on $S_{\alpha}$ using delta functions, leading to the partition functions

$$
\begin{aligned}
& \mathcal{Z}_{\mathrm{D}}=\frac{1}{\mathcal{Z}_{0}} \int \mathcal{D} \Phi \prod_{\alpha=1}^{2} \prod_{X_{\alpha}} \delta\left[\Phi\left(X_{\alpha}\right)\right] \exp \left(-S_{\mathrm{E}}\{\Phi\} / \hbar\right), \\
& \mathcal{Z}_{\mathrm{N}}=\frac{1}{\mathcal{Z}_{0}} \int \mathcal{D} \Phi \prod_{\alpha=1}^{2} \prod_{X_{\alpha}} \delta\left[\partial_{n} \Phi\left(X_{\alpha}\right)\right] \exp \left(-S_{\mathrm{E}}\{\Phi\} / \hbar\right)
\end{aligned}
$$

where $\mathcal{Z}_{0}$ is the partition function of the space without plates. Note that in Eqs. (6a) and 6b) no gauge fixing procedure is needed, since the relevant degrees of freedom are expressed in terms of the fields $E_{2}$ and $B_{2}$ itself rather than the vector potential $A_{\mu}$ in Eq. (2). The details of the calculation of the partition functions are left to Appendix A, and yield

$$
\ln \mathcal{Z}_{\mathrm{D}}=-\frac{1}{2} \operatorname{tr} \ln \Gamma_{\mathrm{D}}, \quad \ln \mathcal{Z}_{\mathrm{N}}=-\frac{1}{2} \operatorname{tr} \ln \Gamma_{\mathrm{N}}
$$

The kernels $\Gamma_{\mathrm{D}}$ and $\Gamma_{\mathrm{N}}$ are given by

$$
\begin{aligned}
{\left[\Gamma_{\mathrm{D}}\right]_{\alpha \beta}\left(\mathbf{y}, \mathbf{y}^{\prime}\right) } & =\left[g_{\alpha}\left(y_{1}\right)\right]^{1 / 4} G\left[X_{\alpha}(\mathbf{y})-X_{\beta}\left(\mathbf{y}^{\prime}\right)\right]\left[g_{\beta}\left(y_{1}^{\prime}\right)\right]^{1 / 4} \\
{\left[\Gamma_{\mathrm{N}}\right]_{\alpha \beta}\left(\mathbf{y}, \mathbf{y}^{\prime}\right) } & =\left[g_{\alpha}\left(y_{1}\right)\right]^{1 / 4} \partial_{n_{\alpha}\left(y_{1}\right)} \partial_{n_{\beta}\left(y_{1}^{\prime}\right)} G\left[X_{\alpha}(\mathbf{y})-X_{\beta}\left(\mathbf{y}^{\prime}\right)\right]\left[g_{\beta}\left(y_{1}^{\prime}\right)\right]^{1 / 4}
\end{aligned}
$$

where $g_{\alpha}\left(y_{1}\right)=1+\left[h_{\alpha}^{\prime}\left(y_{1}\right)\right]^{2}$ is the determinant of the induced metric, and $n_{\alpha}\left(y_{1}\right)=(-1)^{\alpha} g_{\alpha}^{-1 / 2}\left(y_{1}\right)\left[h_{\alpha}^{\prime}\left(y_{1}\right), 0,-1\right]$ is the normal vector to the surface $S_{\alpha}$, while

$$
G(\underline{r})=\frac{1}{4 \pi^{2}} \frac{1}{\underline{r}^{2}}
$$

is the free correlation function (Gaussian propagator) corresponding to the Euclidean action $S_{\mathrm{E}}$ in Eq. (5). In the following, we denote position vectors in the $4 \mathrm{D}$ Euclidean space by $\underline{r}=(\mathbf{y}, z)$. We can then extract the Casimir energy $\mathcal{E}$ per unit area as

$$
\mathcal{E}(H)=E(H)-\lim _{H \rightarrow \infty} E(H)
$$

with

$$
E(H)=-\frac{\hbar c}{A L}\left[\ln \mathcal{Z}_{\mathrm{D}}+\ln \mathcal{Z}_{\mathrm{N}}\right]
$$

where $A$ is the surface area of the plates, and $L$ denotes the overall Euclidean length in time direction.

The above equations provide an exact result which yields the Casimir energy for arbitrary static uniaxial deformations. These equations can be used to evaluate the Casimir force by a recently developed numerical approach [57]. However, in order to obtain a closed analytical expression for the partition functions in terms of the kernels in Eqs. (\$), here we resort to a perturbative expansion with respect to the height profiles $h_{\alpha}\left(y_{1}\right)$. In fact, for this expansion to be valid, we have to assume that the amplitude of the deformations sets the smallest (geometric) length scale of the system. In what follows, perturbation theory is carried out to second order in $h_{\alpha}\left(y_{1}\right)$, separately for the two types of boundary conditions. However, within second order in the height profile, the result is exact in the sense that it correctly takes into account the many-body nature of the Casimir interaction.

\section{A. Dirichlet boundary conditions}

Following Refs. 47, 55, we expand $\ln \mathcal{Z}_{\mathrm{D}}$ in a series $\left.\ln \mathcal{Z}_{\mathrm{D}}\right|_{0}+\left.\ln \mathcal{Z}_{\mathrm{D}}\right|_{1}+\left.\ln \mathcal{Z}_{\mathrm{D}}\right|_{2}+\ldots$, where the subscript indicates the corresponding order in $h_{\alpha}$. The lowest order result is

$$
\left.\ln \mathcal{Z}_{\mathrm{D}}\right|_{0}=\frac{A L}{H^{3}} \frac{\pi^{2}}{1440}
$$


corresponding to two flat plates. The first order result $\left.\ln \mathcal{Z}_{\mathrm{D}}\right|_{1}$ vanishes since we assume, without loss of generality, that the mean deformations are zero, $\int d y_{1} h_{\alpha}\left(y_{1}\right)=0$. The complete second order contribution is given by

$$
\begin{aligned}
\left.\ln \mathcal{Z}_{\mathrm{D}}\right|_{2}= & -\frac{1}{4} \int d^{3} y\left\{\left[h_{1}^{\prime}\left(y_{1}\right)\right]^{2}+\left[h_{2}^{\prime}\left(y_{1}\right)\right]^{2}\right\} \int \frac{d^{3} p}{(2 \pi)^{3}} \times 1 \\
& +\frac{\pi^{2}}{240} \frac{1}{H^{5}} \int d^{3} y\left\{\left[h_{1}\left(y_{1}\right)\right]^{2}+\left[h_{2}\left(y_{1}\right)\right]^{2}\right\} \\
& +\frac{1}{2} \int d^{3} y \int d^{3} y^{\prime} K_{\mathrm{D}}\left(\left|\mathbf{y}-\mathbf{y}^{\prime}\right|\right)\left\{-\frac{1}{2}\left[h_{1}\left(y_{1}\right)-h_{1}\left(y_{1}^{\prime}\right)\right]^{2}-\frac{1}{2}\left[h_{2}\left(y_{1}\right)-h_{2}\left(y_{1}^{\prime}\right)\right]^{2}\right\} \\
& -\frac{1}{2} \int d^{3} y \int d^{3} y^{\prime} Q_{\mathrm{D}}\left(\left|\mathbf{y}-\mathbf{y}^{\prime}\right|\right)\left[h_{1}\left(y_{1}\right) h_{2}\left(y_{1}^{\prime}\right)+h_{2}\left(y_{1}\right) h_{1}\left(y_{1}^{\prime}\right)\right] .
\end{aligned}
$$

In the first term, which is further discussed below, $h_{\alpha}^{\prime}=\nabla h_{\alpha}$. The kernels appearing above are given by [59]

$$
\begin{aligned}
& K_{\mathrm{D}}(y)=F_{1}(y) \partial_{z}^{2} G(y, 0)+F_{1}(y) F_{5}(y)+F_{3}(y)^{2}, \\
& Q_{\mathrm{D}}(y)=F_{4}(y) \partial_{z}^{2} G(y, H)+F_{4}(y) F_{6}(y)+F_{2}(y)^{2}
\end{aligned}
$$

with the set of functions

$$
\begin{aligned}
& F_{1}(y)=\int \frac{d^{3} p}{(2 \pi)^{3}} e^{i \mathbf{p} \cdot \mathbf{y}} \frac{G(p, 0)}{\mathcal{N}(p, H)} \\
& F_{2}(y)=\int \frac{d^{3} p}{(2 \pi)^{3}} e^{i \mathbf{p} \cdot \mathbf{y}} \frac{G(p, 0)}{\mathcal{N}(p, H)} \partial_{z} G(p, H), \\
& F_{3}(y)=\int \frac{d^{3} p}{(2 \pi)^{3}} e^{i \mathbf{p} \cdot \mathbf{y}} \frac{G(p, H)}{\mathcal{N}(p, H)} \partial_{z} G(p, H) \\
& F_{4}(y)=\int \frac{d^{3} p}{(2 \pi)^{3}} e^{i \mathbf{p} \cdot \mathbf{y}} \frac{G(p, H)}{\mathcal{N}(p, H)}, \\
& F_{5}(y)=\int \frac{d^{3} p}{(2 \pi)^{3}} e^{i \mathbf{p} \cdot \mathbf{y}} \frac{G(p, 0)}{\mathcal{N}(p, H)}\left[\partial_{z} G(p, H)\right]^{2}, \\
& F_{6}(y)=\int \frac{d^{3} p}{(2 \pi)^{3}} e^{i \mathbf{p} \cdot \mathbf{y}} \frac{G(p, H)}{\mathcal{N}(p, H)}\left[\partial_{z} G(p, H)\right]^{2},
\end{aligned}
$$

where $y=|\mathbf{y}|, p=|\mathbf{p}|, G(p, z)=\frac{1}{2 p} e^{-p|z|}$ is the partially Fourier transformed free propagator of Eq. (9), and $\mathcal{N}(p, H)=[G(p, 0)]^{2}-[G(p, H)]^{2}$. The functions in Eqs. (15) can be calculated explicitly (see Appendix B 1), leading to the simple result

$$
\begin{aligned}
K_{\mathrm{D}}(y) & =2 K_{\mathrm{D}, \infty}(y)+K_{\mathrm{D}, \mathrm{reg}}(y) \\
Q_{\mathrm{D}}(y) & =\frac{\pi^{2}}{128} \frac{1}{H^{6} y^{2}} \frac{\sinh ^{2}(s)}{\cosh ^{6}(s)}
\end{aligned}
$$

with

$$
K_{\mathrm{D}, \infty}(y)=\frac{1}{2 \pi^{4} y^{8}}, \quad K_{\mathrm{D}, \mathrm{reg}}(y)=-\frac{1}{2 \pi^{4} y^{8}}+\frac{\pi^{2}}{128} \frac{1}{H^{6} y^{2}} \frac{\cosh ^{2}(s)}{\sinh ^{6}(s)},
$$

where $s=\pi y /(2 H)$. The kernel $K_{\mathrm{D}}$ has two contributions of different origin. In the limit $H \rightarrow \infty$, corresponding to two decoupled surfaces, one has $K_{\mathrm{D}} \rightarrow 2 K_{\mathrm{D}, \infty}$, while $Q_{\mathrm{D}}(y)$ vanishes. Thus the part $K_{\mathrm{D}, \infty}$ describes a single surface. The two $\left(H\right.$-independent) single surface contributions have to be subtracted from the total kernel $K_{\mathrm{D}}$ in order to obtain the regularized kernel $K_{\mathrm{D} \text {,reg }}$ which has to be used in the calculation of the Casimir energy in Eq. (10). For 
finite $H$, the kernel $K_{\mathrm{D}}(y)$ actually has contributions from both outside and inside the cavity, whereas $K_{\mathrm{D}, \infty}$ comes from outside and the second term of $K_{\mathrm{D} \text {,reg }}$ from inside. The kernel $Q_{\mathrm{D}}(y)$ has only contributions from inside the cavity.

It is instructive to discuss the meaning of the contributions to $\left.\ln \mathcal{Z}_{\mathrm{D}}\right|_{2}$ in Eq. (13). The terms in the first row are $\mathrm{H}$-independent and formally divergent. They do not contribute to the Casimir force between the surfaces but yield a quantum electrodynamical increase of the surface tension of the individual surfaces after introducing a suitable shortdistance cutoff [60]. The necessity for a cutoff stems from our continuum approach which breaks down on microscopic length scales. The remaining terms in Eq. (13) all contribute to the Casimir force (with $K_{\mathrm{D}}$ replaced by $K_{\mathrm{D}, \mathrm{reg}}$ ). The local contributions in the second row are half (due to TM modes only) of the individual surface (non-mixed) terms which follow in second order of perturbation theory in $h_{\alpha}$ from the pairwise summation approach, cf. the second term in Eq. (26). The third row in Eq. (13) describes non-local individual surface contributions which are missing in the pairwise summation approach. Finally, the last row accounts for contributions due to the interference between the two surface profiles. Obviously, it has a more complicated form than the corresponding last term in the approximative pairwise summation result in Eq. (26).

\section{B. Neumann boundary conditions}

Expanding $\ln \mathcal{Z}_{\mathrm{N}}$ in a series with respect to $h_{\alpha}$ as before, the lowest order result is the same as for the Dirichlet case,

$$
\left.\ln \mathcal{Z}_{\mathrm{N}}\right|_{0}=\frac{A L}{H^{3}} \frac{\pi^{2}}{1440}
$$

and the first order result $\left.\ln \mathcal{Z}_{\mathrm{N}}\right|_{1}$ again vanishes. The complete second order result assumes a similar form as for Dirichlet boundary conditions. We find

$$
\begin{aligned}
\left.\ln \mathcal{Z}_{\mathrm{N}}\right|_{2}= & +\frac{1}{4} \int d^{3} y\left\{\left[h_{1}^{\prime}\left(y_{1}\right)\right]^{2}+\left[h_{2}^{\prime}\left(y_{1}\right)\right]^{2}\right\} \int \frac{d^{3} p}{(2 \pi)^{3}} \times 1 \\
& +\frac{\pi^{2}}{240} \frac{1}{H^{5}} \int d^{3} y\left\{\left[h_{1}\left(y_{1}\right)\right]^{2}+\left[h_{2}\left(y_{1}\right)\right]^{2}\right\} \\
& +\frac{1}{2} \int d^{3} y \int d^{3} y^{\prime} K_{\mathrm{N}}\left(\left|y_{0}-y_{0}^{\prime}\right|,\left|\mathbf{y}_{\|}-\mathbf{y}_{\|}^{\prime}\right|\right)\left\{-\frac{1}{2}\left[h_{1}\left(y_{1}\right)-h_{1}\left(y_{1}^{\prime}\right)\right]^{2}-\frac{1}{2}\left[h_{2}\left(y_{1}\right)-h_{2}\left(y_{1}^{\prime}\right)\right]^{2}\right\} \\
& -\frac{1}{2} \int d^{3} y \int d^{3} y^{\prime} Q_{\mathrm{N}}\left(\left|y_{0}-y_{0}^{\prime}\right|,\left|\mathbf{y}_{\|}-\mathbf{y}_{\|}^{\prime}\right|\right)\left[h_{1}\left(y_{1}\right) h_{2}\left(y_{1}^{\prime}\right)+h_{2}\left(y_{1}\right) h_{1}\left(y_{1}^{\prime}\right)\right] .
\end{aligned}
$$

The kernels for Neumann boundary conditions assume a more complicated form since the normal derivative breaks the equivalence of space and time directions. The result reads

$$
\begin{aligned}
K_{\mathrm{N}}\left(\left|y_{0}\right|,\left|\mathbf{y}_{\|}\right|\right)= & \mathcal{F}_{1}(y) \partial_{z}^{2} g(y, 0)+\mathcal{F}_{1}(y) \mathcal{F}_{5}(y)+\mathcal{F}_{3}(y)^{2} \\
& +\partial_{i} \partial_{j}\left[\mathcal{F}_{1}(y) \partial_{i} \partial_{j} G(y, 0)+\mathcal{F}_{1}(y) \partial_{i} \partial_{j} \mathcal{F}_{7}(y)+\partial_{i} \mathcal{F}_{9}(y) \partial_{j} \mathcal{F}_{9}(y)\right] \\
& +2 \partial_{i}\left[\mathcal{F}_{1}(y) \partial_{i} g(y, 0)+\mathcal{F}_{1}(y) \partial_{i} \mathcal{F}_{11}(y)+\mathcal{F}_{3}(y) \partial_{i} \mathcal{F}_{9}(y)\right] \\
Q_{\mathrm{N}}\left(\left|y_{0}\right|,\left|\mathbf{y}_{\|}\right|\right)= & \mathcal{F}_{4}(y) \partial_{z}^{2} g(y, H)+\mathcal{F}_{4}(y) \mathcal{F}_{6}(y)+\mathcal{F}_{2}(y)^{2} \\
& +\partial_{i} \partial_{j}\left[\mathcal{F}_{4}(y) \partial_{i} \partial_{j} G(y, H)+\mathcal{F}_{4}(y) \partial_{i} \partial_{j} \mathcal{F}_{8}(y)+\partial_{i} \mathcal{F}_{10}(y) \partial_{j} \mathcal{F}_{10}(y)\right] \\
& +2 \partial_{i}\left[\mathcal{F}_{4}(y) \partial_{i} g(y, H)+\mathcal{F}_{4}(y) \partial_{i} \mathcal{F}_{12}(y)+\mathcal{F}_{2}(y) \partial_{i} \mathcal{F}_{10}(y)\right]
\end{aligned}
$$

where summation over $i=1,2$ and $j=1,2$ is understood. Note that $\partial_{i}$ and $\partial_{j}$ act on the spatial components $\mathbf{y}_{\|}$ of $\mathbf{y}$ only. This is the reason why the rotational symmetry within the 3 dimensional $\mathbf{y}$ space is broken for Neumann boundary conditions. For translationally invariant profiles $h_{\alpha}\left(y_{1}\right)$ only terms with $i=j=1$ contribute as can be seen by integration by parts. However, as in the case of Dirichlet boundary conditions, the above result is valid for any $h_{\alpha}(\mathbf{y})$ but then can no longer be interpreted as the contribution from TE modes to the electrodynamic Casimir energy.

Here, we have introduced

$$
g(y, z)=\int \frac{d^{3} p}{(2 \pi)^{3}} e^{i \mathbf{p} \cdot \mathbf{y}} g(p, z)
$$


with $g(p, z)=\partial_{z}^{2} G(p, z)=\frac{p}{2} e^{-p|z|}$, and the functions $\mathcal{F}_{j}(y)$ defined as

$$
\begin{aligned}
& \mathcal{F}_{1}(y)=\int \frac{d^{3} p}{(2 \pi)^{3}} e^{i \mathbf{p} \cdot \mathbf{y}} \frac{g(p, 0)}{\eta(p, H)}, \\
& \mathcal{F}_{2}(y)=\int \frac{d^{3} p}{(2 \pi)^{3}} e^{i \mathbf{p} \cdot \mathbf{y}} \frac{g(p, 0)}{\eta(p, H)} \partial_{z} g(p, H) \\
& \mathcal{F}_{3}(y)=\int \frac{d^{3} p}{(2 \pi)^{3}} e^{i \mathbf{p} \cdot \mathbf{y}} \frac{g(p, H)}{\eta(p, H)} \partial_{z} g(p, H) \\
& \mathcal{F}_{4}(y)=\int \frac{d^{3} p}{(2 \pi)^{3}} e^{i \mathbf{p} \cdot \mathbf{y}} \frac{g(p, H)}{\eta(p, H)} \\
& \mathcal{F}_{5}(y)=\int \frac{d^{3} p}{(2 \pi)^{3}} e^{i \mathbf{p} \cdot \mathbf{y}} \frac{g(p, 0)}{\eta(p, H)}\left[\partial_{z} g(p, H)\right]^{2}, \\
& \mathcal{F}_{6}(y)=\int \frac{d^{3} p}{(2 \pi)^{3}} e^{i \mathbf{p} \cdot \mathbf{y}} \frac{g(p, H)}{\eta(p, H)}\left[\partial_{z} g(p, H)\right]^{2}, \\
& \mathcal{F}_{7}(y)=\int \frac{d^{3} p}{(2 \pi)^{3}} e^{i \mathbf{p} \cdot \mathbf{y}} \frac{g(p, 0)}{\eta(p, H)}\left[\partial_{z} G(p, H)\right]^{2}, \\
& \mathcal{F}_{8}(y)=\int \frac{d^{3} p}{(2 \pi)^{3}} e^{i \mathbf{p} \cdot \mathbf{y}} \frac{g(p, H)}{\eta(p, H)}\left[\partial_{z} G(p, H)\right]^{2}, \\
& \mathcal{F}_{9}(y)=\int \frac{d^{3} p}{(2 \pi)^{3}} e^{i \mathbf{p} \cdot \mathbf{y}} \frac{g(p, H)}{\eta(p, H)} \partial_{z} G(p, H), \\
& \mathcal{F}_{10}(y)=\int \frac{d^{3} p}{(2 \pi)^{3}} e^{i \mathbf{p} \cdot \mathbf{y}} \frac{g(p, 0)}{\eta(p, H)} \partial_{z} G(p, H), \\
& \mathcal{F}_{11}(y)=\int \frac{d^{3} p}{(2 \pi)^{3}} e^{i \mathbf{p} \cdot \mathbf{y}} \frac{g(p, 0)}{\eta(p, H)} \partial_{z} g(p, H) \partial_{z} G(p, H), \\
& \mathcal{F}_{12}(y)=\int \frac{d^{3} p}{(2 \pi)^{3}} e^{i \mathbf{p} \cdot \mathbf{y}} \frac{g(p, H)}{\eta(p, H)} \partial_{z} g(p, H) \partial_{z} G(p, H),
\end{aligned}
$$

with $\eta(p, H)=[g(p, 0)]^{2}-[g(p, H)]^{2}$. The explicit form of these functions can be found in Appendix B2.

The result in Eq. (19) has the same type of contributions as discussed for the Dirichlet case. Both Dirichlet and Neumann case include 'surface tension' contributions, but with opposite signs, and identical local terms (second row in Eq. (19)). Since these local terms are the only (non-mixed) contributions obtained by the pairwise summation approach, the latter does not distinguish between the two types of boundary conditions. The main results of our general analysis of surface deformations are contained in Eqs. 130, 19]. In section IV we apply these results to the important case of modulated plates.

\section{PAIRWISE SUMMATION APPROXIMATION}

The path integral approach may be compared with the commonly used approximative method of pairwise summation (PWS). In the latter approach, the Casimir energy $\mathcal{E}(H)$ for two arbitrary shaped bodies of mean distance $H$ is obtained 
by the pairwise summation of a two-body potential $U(r)$. In terms of the deformation fields $h_{\alpha}$ this leads to

$$
\mathcal{E}(H)=\frac{1}{A} \int d^{2} \mathbf{y}_{\|} \int d^{2} \mathbf{y}_{\|}^{\prime} \int_{H+h_{2}\left(y_{1}\right)}^{\infty} d z \int_{-\infty}^{h_{1}\left(y_{1}\right)} d z^{\prime} U\left[\left(\left(\mathbf{y}_{\|}-\mathbf{y}_{\|}^{\prime}\right)^{2}+\left(z-z^{\prime}\right)^{2}\right)^{1 / 2}\right] .
$$

In general, these integrals need to be computed numerically. However, there are the following simplifications. If one of the plates is flat, e.g., $h_{1}\left(y_{1}\right)=0$, the integrals can be performed explicitly, leading to the simple result

$$
\mathcal{E}(H)=\frac{1}{A} \int d^{2} \mathbf{y}_{\|} \mathcal{E}_{0}\left[H+h_{2}\left(y_{1}\right)\right]
$$

where $\mathcal{E}_{0}$ is here the energy of two flat plates at distance $H$, calculated from the same pair potential $U(r)$. Thus, in this particular case the pairwise summation approximation is equivalent to a geometrical average of the flat plate energy with locally varying plate distance over the plate area.

For two deformed plates, the integrals in Eq. (23) in general can only be performed perturbatively in the height profile. To do so, we follow the usual PWS approximation and assume a 'renormalized' retarded van der Waals potential [11, 58],

$$
U(r)=-\frac{\pi \hbar c}{24} r^{-7}
$$

The 'renormalization factor' of the pair potential is chosen here such that in the limit of two flat plates the exact Casimir result $\mathcal{E}_{0}$, cf. Eq. (32), is recovered. To second order in $h_{\alpha}$ one obtains now

$$
\mathcal{E}(H)=-\frac{\pi^{2}}{720} \frac{\hbar c}{H^{3}}-\frac{\pi^{2}}{120} \frac{\hbar c}{H^{5} A} \int d^{2} \mathbf{y}_{\|}\left[h_{1}^{2}\left(y_{1}\right)+h_{2}^{2}\left(y_{1}\right)\right]+\frac{\pi}{24} \frac{\hbar c}{A} \int d^{2} \mathbf{y}_{\|} \int d^{2} \mathbf{y}_{\|}^{\prime} \frac{h_{1}\left(y_{1}+y_{1}^{\prime}\right) h_{2}\left(y_{1}\right)}{\left(H^{2}+\mathbf{y}_{\|}^{\prime 2}\right)^{7 / 2}}
$$

For simple types of plate modulations, the integrals over the deformation fields can be calculated easily. This will allow us a direct assessment of the validity range of the pairwise summation approach by comparing Eq. (26) to the predictions of the path integral technique.

\section{MODULATED PLATES}

We now apply the results of Sec. II to static uniaxial modulations of two parallel plates. In the first part we focus on the normal Casimir force between a flat and a corrugated plate. This force per unit area is defined as

$$
F_{\mathrm{n}}=-\frac{\partial \mathcal{E}}{\partial H}
$$

in terms of the Casimir energy in Eq. (10). However, in most of the experiments, the flat plate is replaced by a spherical lens with large radius $R \gg H$. In the latter case the normal force can be obtained by using the Derjaguin approximation (DA) (or proximity force rule) 22, leading to

$$
F_{\mathrm{DA}, \mathrm{n}}=2 \pi R \mathcal{E} .
$$

Therefore, in the context of the normal Casimir force, we just calculate $\mathcal{E}$ explicitly.

In the second part of this section we generalize our results for two modulated plates with equal modulation length but with a phase shift between them. Due to the broken translational symmetry, there is now also a lateral force between the two plates which arises solely from the cross-terms $\sim h_{1} h_{2}$ in Eqs. (13), (19). If we denote the shift between the two corrugations by the length $b$, the lateral force is obtained from

$$
F_{1}=-\frac{\partial \mathcal{E}}{\partial b}
$$

\section{A. Normal force}

As a prototype of a corrugated surface, and to make contact with recent experiments by Roy and Mohideen [49, we consider a sinusoidally modulated plate along the $y_{1}$ direction, with amplitude $a$, wavelength $\lambda$, and mean distance $H$ from the flat plate (see Fig. 1), i.e.,

$$
h_{1}\left(y_{1}\right)=a \cos \left(2 \pi y_{1} / \lambda\right), \quad \text { and } \quad h_{2}\left(y_{1}\right)=0 .
$$




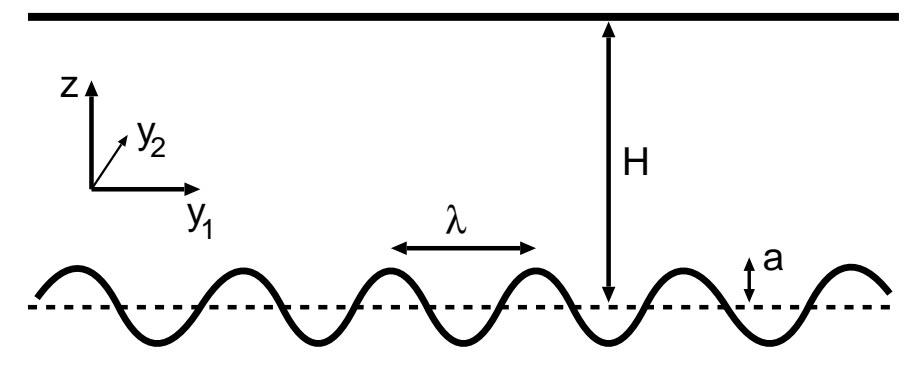

FIG. 1: The set-up used for calculating the Casimir energy between a flat and a corrugated plate at mean separation $H$.

For this particular deformation [50], only the Fourier mode of wavelength $\lambda$ in the kernels $K_{\mathrm{D}}(y)$ and $K_{\mathrm{N}}\left(\left|y_{0}\right|,\left|\mathbf{y}_{\|}\right|\right)$ is probed. Thus, the calculation of $\ln \mathcal{Z}_{\mathrm{D}}$ and $\ln \mathcal{Z}_{\mathrm{N}}$ via Eqs. (13), (19) reduces to Fourier transforming the kernels with respect to $y_{1}$. The corresponding expression for $\mathcal{E}$ in Eq. (10) can be written as

$$
\mathcal{E}=\mathcal{E}_{0}+\mathcal{E}_{\mathrm{cf}}
$$

with

$$
\mathcal{E}_{0}=-\frac{\pi^{2}}{720} \frac{\hbar c}{H^{3}}, \quad \mathcal{E}_{\mathrm{cf}}=-\frac{\hbar c a^{2}}{H^{5}}\left[G_{\mathrm{TM}}\left(\frac{H}{\lambda}\right)+G_{\mathrm{TE}}\left(\frac{H}{\lambda}\right)\right]+\mathcal{O}\left(a^{3}\right),
$$

where $\mathcal{E}_{0}$ is the energy of two flat plates and the index cf of $\mathcal{E}_{\text {cf }}$ stands for corrugated-flat geometry. The notation $\mathcal{O}\left(a^{3}\right)$ indicates that third and higher powers of $a / H$ and $a / \lambda$ are not considered here. The corrugation induced contributions to $\mathcal{E}_{\text {cf }}$ from TM and TE modes at second order in $a$ are governed by the functions

$$
\begin{aligned}
& G_{\mathrm{TM}}(x)=\frac{\pi^{2}}{480}+g_{0}(x), \\
& G_{\mathrm{TE}}(x)=\frac{\pi^{2}}{480}+g_{1}(x)+x g_{2}(x)+x^{3} g_{3}(x) .
\end{aligned}
$$

The first term $\pi^{2} / 480$ in both equations corresponds to the local contributions, cf. the second row in Eqs. (13), (19). Since these are the only terms which are obtained within a pairwise summation approach, the functions $g_{m}(x)$ represent non-trivial corrections which are neglected in the pairwise summation scheme. These functions can be 
calculated from the kernels $K_{\mathrm{D}}(y)$ and $K_{\mathrm{N}}\left(\left|y_{0}\right|,\left|\mathbf{y}_{\|}\right|\right)$by Fourier transformation, leading to the expressions

$$
\begin{aligned}
g_{0}(x)= & -\frac{\pi^{2}}{480}+\frac{\pi^{3}}{480} x+\frac{\pi^{2}}{128} \int_{-\infty}^{\infty} d s \frac{\sin (4 x s)}{4 x s}\left[-\frac{1}{s^{6}}+\frac{2}{15 s^{2}}+\frac{\cosh ^{2}(s)}{\sinh ^{6}(s)}\right], \\
g_{1}(x)= & -\frac{\pi^{2}}{480}+\frac{\pi^{3}}{1440} x \\
& +\frac{\pi^{2}}{64} \int_{-\infty}^{\infty} d s \frac{\sin (4 x s)}{4 x s}\left[-\frac{13}{4 s^{6}}-\frac{5}{3 s^{4}}+\frac{4}{45 s^{2}}+\frac{5}{2 s^{5}} \frac{\cosh (s)}{\sinh (s)}-\frac{3}{2 s^{3}} \frac{\cosh (s)}{\sinh ^{3}(s)}\right. \\
& \left.+\frac{1}{2 s} \frac{\cosh ^{3}(s)}{\sinh ^{5}(s)}+\frac{1}{s} \frac{\cosh (s)}{\sinh ^{5}(s)}+\frac{1}{2} \frac{\cosh ^{4}(s)}{\sinh ^{6}(s)}+\frac{5}{4} \frac{\cosh ^{2}(s)}{\sinh ^{6}(s)}-\frac{1}{s^{4}} \frac{1}{\sinh ^{2}(s)}\right] \\
& -\frac{\pi^{2}}{64} \int_{-\infty}^{\infty} d s \cos (4 x s)\left[\frac{1}{s^{6}}+\frac{1}{45 s^{2}}-\frac{2}{3 s^{4}}-\frac{1}{s^{4}} \frac{1}{\sinh ^{2}(s)}+\frac{1}{s^{5}} \frac{\cosh (s)}{\sinh (s)}-\frac{1}{s^{3}} \frac{\cosh (s)}{\sinh ^{3}(s)}\right], \\
g_{2}(x)= & \frac{\pi^{2}}{64} \int_{-\infty}^{\infty} d s \sin (4 x s)\left[\frac{2}{45 s}-\frac{5}{s^{5}}+\frac{1}{s^{4}} \frac{\cosh (s)}{\sinh (s)}\right. \\
& \left.+\frac{2}{s^{3}} \frac{1}{\sinh (s)}+\frac{1}{s^{2}} \frac{\cosh ^{2}(s)}{\sinh ^{3}(s)}+\frac{1}{s} \frac{\cosh ^{2}(s)}{\sinh ^{4}(s)}\right], \\
g_{3}(x)= & \frac{\pi}{32} \int_{-\infty}^{\infty} d s \sin (4 x s)\left[-\frac{3}{s^{3}}-\frac{4}{3 s}+\frac{2}{s^{2}} \frac{\cosh (s)}{\sinh (s)}+\frac{1}{s} \frac{\cosh ^{2}(s)}{\sinh ^{2}(s)}\right] .
\end{aligned}
$$

Before giving the explicit forms of these functions, let us consider two limiting cases. For $x \rightarrow 0$, or $\lambda / H \rightarrow \infty$, we have $g_{0}(x), g_{1}(x) \rightarrow 0$, and $g_{2}(x), g_{3}(x)$ converge to finite numbers, thus leaving in Eqs. (33) only the local contributions from the pairwise summation approach. In the opposite limit $x \rightarrow \infty$, or $\lambda / H \rightarrow 0$, the integrals in Eqs. (34) decay to zero, leading to

$$
g_{0}(x)=\frac{\pi^{2}}{480}\left(\pi x-1+\frac{5 \pi}{126} \frac{1}{x}\right)+\mathcal{O}\left(e^{-4 \pi x}\right), \quad g_{1}(x)=\frac{\pi^{2}}{480}\left(\frac{\pi}{3} x-1+\frac{\pi}{18} \frac{1}{x}\right)+\mathcal{O}\left(e^{-4 \pi x}\right),
$$

and both $g_{2}(x)$ and $g_{3}(x)$ are $\mathcal{O}\left(e^{-4 \pi x}\right)$. From this result it is obvious that for $\lambda / H \rightarrow 0$ in Eqs. (33), the terms $\pi^{2} / 480$ from the pairwise summation approach are exactly canceled by corresponding terms of opposite sign in the non-trivial corrections described by $g_{0}(x)$ and $g_{1}(x)$. The most relevant contributions in this limit are now provided by the first term in $g_{0}(x)$ and $g_{1}(x)$ in Eq. (35), leading to the novel scaling behavior $G_{\mathrm{TM} / \mathrm{TE}}(x) \sim x+\mathcal{O}(1 / x)$.

The integrals in Eqs. (34) can be carried out for $\lambda>0$, or equivalently $x>0$, by closing the integration contour via a semi-circle at infinity in the upper half of the complex $s$ plane, using the residue theorem [61]. The resulting sum of an infinite series of residues can be expressed in terms of the polylogarithm function 662]

$$
\operatorname{Li}_{n}(z)=\sum_{\nu=1}^{\infty} \frac{z^{\nu}}{\nu^{n}},
$$

leading to, with $u \equiv \exp (-4 \pi x)$,

$$
\begin{aligned}
G_{\mathrm{TM}}(x)= & \frac{\pi^{3} x}{480}-\frac{\pi^{2} x^{4}}{30} \ln (1-u)+\frac{\pi}{1920 x} \operatorname{Li}_{2}(1-u)+\frac{\pi x^{3}}{24} \mathrm{Li}_{2}(u)+\frac{x^{2}}{24} \mathrm{Li}_{3}(u)+\frac{x}{32 \pi} \operatorname{Li}_{4}(u) \\
& +\frac{1}{64 \pi^{2}} \mathrm{Li}_{5}(u)+\frac{1}{256 \pi^{3} x}\left(\operatorname{Li}_{6}(u)-\frac{\pi^{6}}{945}\right), \\
G_{\mathrm{TE}}(x)= & \frac{\pi^{3} x}{1440}-\frac{\pi^{2} x^{4}}{30} \ln (1-u)+\frac{\pi}{1920 x} \operatorname{Li}_{2}(1-u)-\frac{\pi x}{48}\left(1+2 x^{2}\right) \operatorname{Li}_{2}(u)+\left(\frac{x^{2}}{48}-\frac{1}{64}\right) \mathrm{Li}_{3}(u)+ \\
& +\frac{5 x}{64 \pi} \operatorname{Li}_{4}(u)+\frac{7}{128 \pi^{2}} \operatorname{Li}_{5}(u)+\frac{1}{256 \pi^{3} x}\left(\frac{7}{2} \operatorname{Li}_{6}(u)-\pi^{2} \operatorname{Li}_{4}(u)+\frac{\pi^{6}}{135}\right) .
\end{aligned}
$$

It should be noted that the appearance of the polylogarithm function in quantum electrodynamics is also known from the fine structure constant dependent corrections to the gyromagnetic ratio of the electron [63]. 
Figure 2 displays separately the contributions from $G_{\mathrm{TM}}$ and $G_{\mathrm{TE}}$ to the corrugation induced correction $\mathcal{E}_{\mathrm{cf}}$ to the Casimir energy. While $G_{\mathrm{TM}}(H / \lambda)$ is a monotonically increasing function of $H / \lambda, G_{\mathrm{TE}}(H / \lambda)$ displays a minimum for $H / \lambda \approx 0.3$

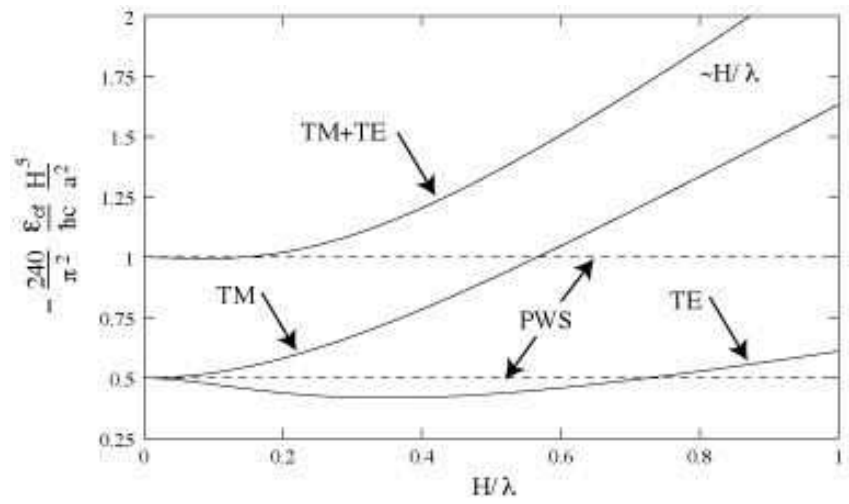

FIG. 2: Rescaled correction $\mathcal{E}_{\text {cf }}$ to the Casimir energy due to the corrugation as given by the terms in square brackets of Eq. (31) (upper curve). The lower curves show the separate contributions from TM and TE modes. The rescaling of $\mathcal{E}_{\mathrm{cf}}$ is chosen such that the corresponding prediction of the pairwise summation (PWS) approximation [second term of Eq. (26)] is a constant (dashed lines).

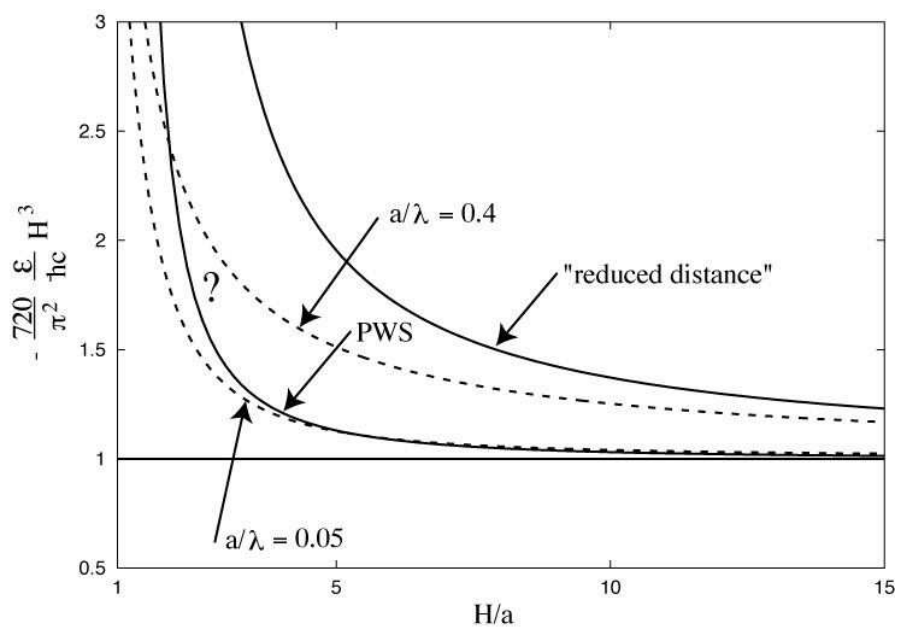

FIG. 3: Rescaled Casimir energy to second order in $a / H$ and $a / \lambda$ as given by Eq. 31 for two fixed values of $a / \lambda$, shown as dashed curves. The rescaling is chosen such that the Casimir energy of two flat plates becomes one (horizontal line). The curve for $a / \lambda=0.05$ corresponds to the parameters used in the experiment of Ref. 49], where $H / a$ varies between approximately 3 and 17. Note that the dashed lines are accurate predictions for the true Casimir energy in the limit $a \ll H, \lambda$ only, as indicated by the question mark (see text and the discussion in Sec.V). For comparison, the result of the pairwise summation (PWS) approximation [cf. Eq. (24)] is shown. It agrees with the perturbative result in the limit $\lambda=\infty$ only. In the opposite limit, $\lambda / a \rightarrow 0$, the energy can presumably be estimated from a reduced distance argument 48, 57] (see Eq. (41)).

Examining the limiting behaviors of Eq. (31) is instructive. In the limit $\lambda \gg H$, the functions $G_{\mathrm{TM}}$ and $G_{\mathrm{TE}}$ approach constant values, and the Casimir energy takes the $\lambda$-independent form

$$
\mathcal{E}=-\frac{\hbar c}{H^{3}} \frac{\pi^{2}}{720}\left(1+3 \frac{a^{2}}{H^{2}}\right)+\mathcal{O}\left(a^{3}\right)
$$

Note that only in this case both wave types provide the same contribution to the total energy and the result agrees with the pairwise summation approximation (see Fig. 2). In the opposite limit of $\lambda \ll H$, as demonstrated above, both 
$G_{\mathrm{TM}}$ and $G_{\mathrm{TE}}$ grow linearly in $H / \lambda$. Therefore, in this limit the correction to the Casimir energy decays according to a slower power law in $H$, as

$$
\mathcal{E}=-\frac{\hbar c}{H^{3}} \frac{\pi^{2}}{720}\left(1+2 \pi \frac{a^{2}}{\lambda H}\right)+\mathcal{O}\left(a^{3}\right),
$$

with an amplitude proportional to $1 / \lambda$. Note that this behavior is completely missed by the pairwise summation approach which always yields a $\lambda$ independent Casimir energy in the presence of modulations on one plate.

In the limit $\lambda \ll a,(H-a)$ we expect that the factor multiplying $a / H$ in Eq. (40) saturates at a number of order unity. This result can be justified by noting that the most relevant contributions to the force come from modes of wavelength of order $H$. The corrugation also affects modes of wavelength of order $\lambda$, but these modes contribute to the single plate energy only. Thus, in the extreme limit $\lambda \ll a,(H-a)$, one has a clear separation of the length scales $H$ and $\lambda$, and the modes "see" flat plates at the reduced separation $H-a,(a>0)$ 48. More recently, an exact approach for calculating the Casimir force has been developed which confirms the above argument and yields for the case of TE modes (Dirichlet boundary conditions at both plates) the exact result [57],

$$
\mathcal{E}=-\frac{\pi^{2}}{720} \frac{\hbar c}{(H-a)^{3}}, \quad \lambda \ll a,(H-a) .
$$

This leads to a correction of the order $a / H$ (with prefactor 3) after expansion in $a$.

The above behavior of the correction $\mathcal{E}_{\mathrm{cf}}$ for small and large $H / \lambda$ clarifies the limits of validity of previous results in the literature. The upper dashed line in Fig. 2 corresponds to the PWS approximation (see Refs. [11, 58] and Sec. [III). It is evident that this approximation is accurate only for $H / \lambda \rightarrow 0$ (which in this limit is equivalent to the Derjaguin method to any order in the amplitude $a$ [22]). Already for $H / \lambda$ of order unity, the PWS approximation breaks down. The opposite limit, $H / \lambda \rightarrow \infty$, corroborates the result reported in Ref. 17, which is larger than the former by a factor of $H / \lambda \gg 1$. However, in experiments with lateral distortions $\lambda$ of the order of $H$, none of the above limiting cases is realized, which makes the present, more complete analysis necessary.

The use of a spherical tip, of large radius $R$, in experiments [49] causes some differences from the flat plate geometry used in our calculations. First, the positioning of the tip relative to the modulations is important when $H$ and $\lambda$ are comparable, but becomes insignificant in the proposed limit of $\lambda \ll H, R$. Secondly, as long as $R \gg H, \lambda$ the curvature of the tip does not lead to nontrivial corrections, and the force can be related to the energy per surface area $\mathcal{E}$ in Eq. (31) by the proximity force rule $F=2 \pi R \mathcal{E}$ in Eq. (28). These formulas thus provide a specific recipe for evaluating the nontrivial shape dependences of the Casimir force in the experimental set-up.

The net Casimir energy $\mathcal{E}$ is shown in Fig. 3 for two representative values of $a / \lambda$, including the parameters used in the experiment of Ref. [49]. Note that the corrugation induced correction leads to a larger energy $\mathcal{E}$, and hence the corresponding (attractive) force $F=2 \pi R \mathcal{E}$ is enhanced, at least to second order in $a / H$ and $a / \lambda$, which becomes exact in the limit $a \ll H, \lambda$. This trend suggests, in particular, that in the set-up of Fig. 1 the force is always attractive, although definite statements for values of $a / H$ and $a / \lambda$ of order one can only be made by using non-perturbative methods, as indicated by the question mark in Fig. 3 (see Ref. [57] and the discussion in Sec. V). However, in the opposite limit, for which the tips of the modulations of the lower plate in Fig. 1 almost touch the upper (flat) plate, i.e., $H-a \ll \lambda^{2} / a, a$, the energy can be calculated exactly by using the Derjaguin approximation for the individual tips of the modulations; this leads to

$$
\mathcal{E}=-\frac{\pi^{2} \sqrt{2}}{3840} \frac{\hbar c}{a^{1 / 2}(H-a)^{5 / 2}}, \quad H-a \rightarrow 0,
$$

which corresponds to the result in Eq. (24) after taking $H-a \rightarrow 0$. The above result implies that at least for the particular case of an uniaxial sinusoidal corrugation the corresponding force $F=2 \pi R \mathcal{E}$ is attractive when the surfaces almost touch.

\section{B. Lateral force}

As a natural generalization of the geometry of the previous section, we study the Casimir interaction between two sinusoidally corrugated plates. For direct correspondence to recent experiments [52], we consider the specific profiles

$$
h_{1}\left(y_{1}\right)=a \cos \left(2 \pi y_{1} / \lambda\right), \quad \text { and } \quad h_{2}\left(y_{1}\right)=a \cos \left(2 \pi\left(y_{1}+b\right) / \lambda\right),
$$

which are shifted relative to each other by the length $b$ (see Fig. 苂). 


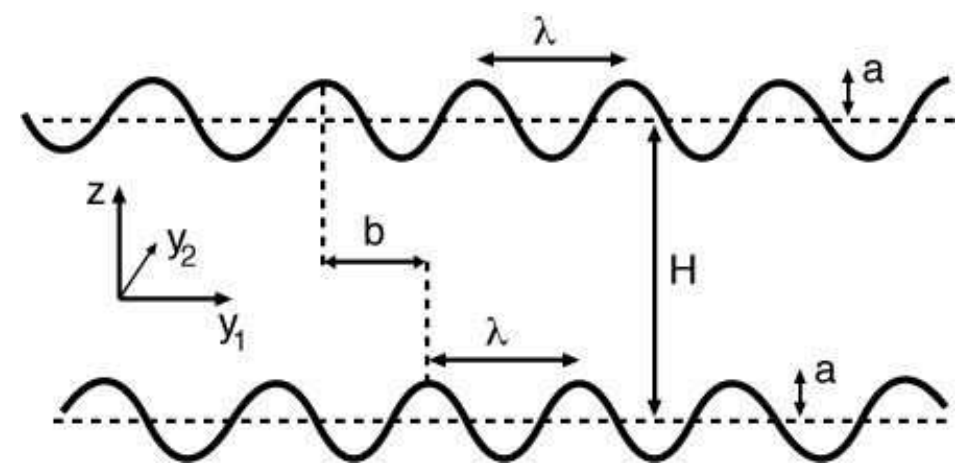

FIG. 4: Geometry used for calculating the lateral Casimir force between two corrugated plates with lateral shift $b$. The equilibrium position is at $b=\lambda / 2$.

By inspection of the deformation dependent contributions to the Casimir energy in Eqs. (13) and (19), one obtains for the total Casimir energy $\mathcal{E}$ of the corrugated-corrugated geometry the relation

$$
\mathcal{E}=\mathcal{E}_{0}+2 \mathcal{E}_{\mathrm{cf}}+\mathcal{E}_{\mathrm{cc}}
$$

with $\mathcal{E}_{0}$ and $\mathcal{E}_{\text {cf }}$ given in Eq. (32), and the corrugation-corrugation interaction energy $\mathcal{E}_{\text {cc }}$ which can be calculated in terms of the kernels $Q_{\mathrm{D}}(y)$ and $Q_{\mathrm{N}}\left(\left|y_{0}\right|,\left|\mathbf{y}_{\|}\right|\right)$in Eqs. (14b) and (20b). Besides oscillating contributions to the normal Casimir force $F_{\mathrm{n}}(b)$ from $\mathcal{E}_{\mathrm{cc}}(b)$, a lateral force

$$
F_{1}=-\frac{\partial \mathcal{E}_{\mathrm{cc}}}{\partial b}
$$

is induced by the corrugation-corrugation interaction. This lateral force is much better suited for experimental tests of the influence of deformations, since there is no need for subtracting a larger baseline force (the contribution of flat plates) as in the case of the normal force.

The calculation of the interaction energy $\mathcal{E}_{\mathrm{cc}}$ again reduces for sinusoidally corrugated plates to Fourier transforming the kernels $Q_{\mathrm{D}}(y)$ and $Q_{\mathrm{N}}\left(\left|y_{0}\right|,\left|\mathbf{y}_{\|}\right|\right)$. Separating the contributions from TM and TE modes, we find

$$
\mathcal{E}_{\mathrm{cc}}=\frac{\hbar c a^{2}}{H^{5}} \cos \left(\frac{2 \pi b}{\lambda}\right)\left[J_{\mathrm{TM}}\left(\frac{H}{\lambda}\right)+J_{\mathrm{TE}}\left(\frac{H}{\lambda}\right)\right]+\mathcal{O}\left(a^{3}\right),
$$

with

$$
\begin{aligned}
J_{\mathrm{TM}}(x) & =j_{0}(x), \\
J_{\mathrm{TE}}(x) & =j_{1}(x)-x^{2} j_{2}(x)+x^{4} j_{3}(x),
\end{aligned}
$$

and the functions $j_{m}(x)$ are given by

$$
\begin{aligned}
& j_{0}(x)=\frac{\pi^{2}}{32} \int_{-\infty}^{\infty} d s \frac{\sin (4 x s)}{4 x s} \frac{\sinh ^{2}(s)}{\cosh ^{6}(s)}, \\
& j_{1}(x)=\frac{\pi^{2}}{32} \int_{-\infty}^{\infty} d s \frac{\sin (4 x s)}{4 x s} \frac{\sinh ^{2}(s)}{\cosh ^{6}(s)}\left[\frac{5}{2}-\sinh ^{2}(s)\right], \\
& j_{2}(x)=\frac{\pi^{2}}{4} \int_{-\infty}^{\infty} d s \frac{\sin (4 x s)}{4 x s} \frac{\sinh ^{2}(s)}{\cosh ^{4}(s)} \\
& j_{3}(x)=\frac{\pi^{2}}{2} \int_{-\infty}^{\infty} d s \frac{\sin (4 x s)}{4 x s} \frac{\sinh ^{2}(s)}{\cosh ^{2}(s)} .
\end{aligned}
$$

Before giving explicit forms for these integrals, it is instructive to again consider their extreme limits. For $x=H / \lambda \rightarrow$ 0 , we find that both functions $J_{\mathrm{TM}}(x)$ and $J_{\mathrm{TE}}(x)$ tend to $\pi^{2} / 240$. In the opposite limit $x=H / \lambda \rightarrow \infty$ both functions 


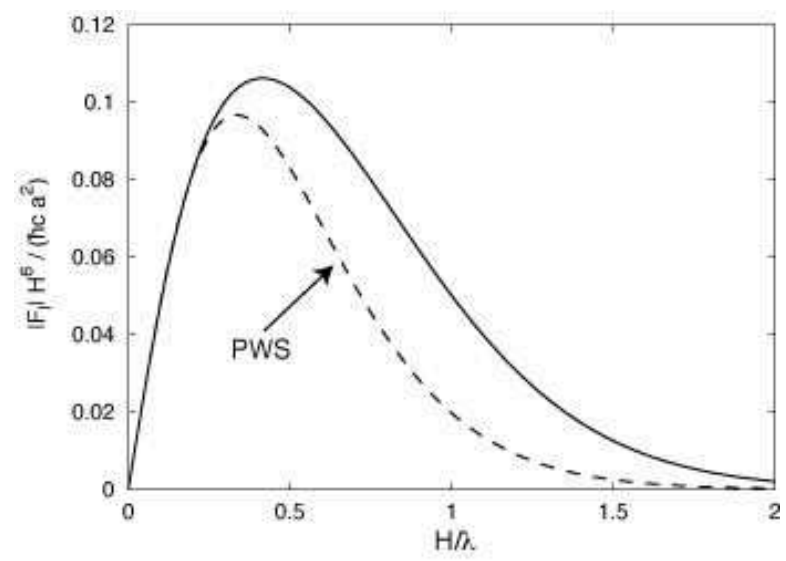

FIG. 5: Rescaled lateral Casimir force amplitude $\left|F_{1}\right|$ as obtained from the path integral approach [Eq. (50)] (solid curve), and from the pairwise summation approach (PWS) [Eq. (51)] (dashed line). The results hold to second order in $a$ (cf. the discussion in Sec. V).

decay exponentially fast to zero so that the lateral force vanishes in this limit. In order to get the behavior in between these extremes, we have to calculate the integrals in Eqs. (48). Using the residue theorem, we finally obtain after summing over an infinite series of residues by using the Lerch transcendent 62

$$
\Phi(z, s, a)=\sum_{k=0}^{\infty} \frac{z^{k}}{(a+k)^{s}}
$$

the results (with $u \equiv \exp (-4 \pi x)$ )

$$
\begin{aligned}
J_{\mathrm{TM}}(x)= & \frac{\pi^{2}}{120}\left(16 x^{4}-1\right) \operatorname{arctanh}(\sqrt{u})+\sqrt{u}\left[\frac{\pi}{12}\left(x^{3}-\frac{1}{80 x}\right) \Phi\left(u, 2, \frac{1}{2}\right)+\frac{x^{2}}{12} \Phi\left(u, 3, \frac{1}{2}\right)\right. \\
& \left.+\frac{x}{16 \pi} \Phi\left(u, 4, \frac{1}{2}\right)+\frac{1}{32 \pi^{2}} \Phi\left(u, 5, \frac{1}{2}\right)+\frac{1}{128 \pi^{3} x} \Phi\left(u, 6, \frac{1}{2}\right)\right], \\
J_{\mathrm{TE}}(x)= & \frac{\pi^{2}}{120}\left(16 x^{4}-1\right) \operatorname{arctanh}(\sqrt{u})+\sqrt{u}\left[-\frac{\pi}{12}\left(x^{3}+\frac{x}{2}+\frac{1}{80 x}\right) \Phi\left(u, 2, \frac{1}{2}\right)+\frac{1}{24}\left(x^{2}-\frac{3}{4}\right) \Phi\left(u, 3, \frac{1}{2}\right)\right. \\
& \left.+\frac{5}{32 \pi}\left(x-\frac{1}{20 x}\right) \Phi\left(u, 4, \frac{1}{2}\right)+\frac{7}{64 \pi^{2}} \Phi\left(u, 5, \frac{1}{2}\right)+\frac{7}{256 \pi^{3} x} \Phi\left(u, 6, \frac{1}{2}\right)\right] .
\end{aligned}
$$

This result can be compared to the pairwise summation approach by considering the last term in Eq. (26). For the surface profiles considered here (cf. Eq. (43)), this term provides an interaction energy given by Eq. (46) with the sum $J_{\mathrm{TM}}(x)+J_{\mathrm{TE}}(x)$ replaced by the function

$$
J_{\mathrm{PWS}}(x)=\frac{\pi^{2}}{360}\left(4 \pi^{2} x^{2}+6 \pi x+3\right) \sqrt{u} .
$$

The two results agree for $x=H / \lambda \rightarrow 0$, since $J_{\mathrm{TM}}(0)+J_{\mathrm{TE}}(0)=\pi^{2} / 120=J_{\mathrm{PWS}}(0)$. At the other extreme of $\lambda \ll H$, both $J_{\mathrm{TM}}(x)+J_{\mathrm{TE}}(x)$ and $J_{\mathrm{PWS}}(x)$ decay exponentially fast but with different $H / \lambda$ dependent coefficients. In particular, for large $x=H / \lambda$, we get to leading order

$$
J_{\mathrm{TM}}(x)+J_{\mathrm{TE}}(x)=\frac{4 \pi^{2}}{15}\left(x^{4}+\mathcal{O}\left(x^{2}\right)\right) \sqrt{u} \quad(x \rightarrow \infty),
$$

in contrast to the $\sim x^{2}$ behavior in Eq. (51).

Since both $J_{\mathrm{TM}}(x)+J_{\mathrm{TE}}(x)$ and $J_{\mathrm{PWS}}(x)$ are positive for all values of $x$, the equilibrium position of two modulated surfaces is predicted at $b=\lambda / 2$ in both approaches. This corresponds to aligning the maxima and minima of the two corrugations (cf. Fig. (1). The amplitude $\left|F_{1}\right|$ of the lateral force per unit area [Eq. (45)] as obtained from both 
approaches is plotted in Fig. 5. Interestingly, for fixed $H$ there is an optimal modulation length $\lambda \approx 2.5 H$ at which the lateral force is largest. Our result shows that the pairwise summation approximation is not justified beyond $H / \lambda \approx 0.3$. For $H / \lambda$ of order one, the pairwise summation approach has already a relative error of about $150 \%$. With increasing $H / \lambda$ this error grows monotonically.

\section{DISCUSSION AND OUTLOOK}

We calculated normal and lateral Casimir forces between perfectly conducting modulated plates (Figs. 1 and 4) by means of the path integral quantization method (see Refs. 47, 55, and Sec. III). Based on the resulting exact expressions for the Casimir energy (Eqs. (7) - (11)), we performed a perturbative calculation to second order in the deformation parameter $a$ to obtain the results outlined in Sec. IV and shown in Figs. 2 and 3 (normal force) and Fig. 5 (lateral force). These results are thus exact to second order in $a$, and correctly take into account the many-body nature of the Casimir interaction, going beyond the commonly used pairwise summation (PWS) of van der Waals forces [11, 58]. Our results show significant deviations from PWS to second order in $a$. However, for finite values of $a / \lambda$ and $a / H$, there will be corrections at higher orders in $a / \lambda$ and $a / H$; in the present experiments, the sensitive range of $a / H$ is of the order of 0.2 [49, 52, while we suggest values for $a / \lambda$ of order one to probe the nontrivial shape dependence of the Casimir force (see below). At present, it is not clear how relevant the perturbative results to second (or any finite) order in $a$ are for this range of the parameters $a / \lambda$ and $a / H$ (cf. the related discussion in Sec. IV A).

To make further connection between our findings and the experimental situations, corrections due to the finite conductivity of the plates, finite temperature, and surface roughness should be taken into account as well 11, 17, 18, 19, 20, 21. These corrections introduce additional length scales into the problem, which are in turn the plasma wavelength $\lambda_{p}$ of the plates (e.g., $\lambda_{p} \approx 100 \mathrm{~nm}$ for aluminum [49]), the thermal wavelength $\lambda_{T}=\hbar c / k_{B} T(\approx 8 \mu \mathrm{m}$ at $300^{\circ} \mathrm{K}$ ), and the transverse correlation length $\xi$ of the roughness (usually $\xi \approx 300 \mathrm{~nm}[16]$ ). While the effect of finite conductivity becomes important for $H \lesssim \lambda_{p}$, finite temperature starts to affect the result when $H \gtrsim \lambda_{T}$. The plasma and thermal wavelengths thus provide lower and upper bounds for $H$, respectively, such that our results for perfectly conducting plates at zero temperature are valid for $\lambda, H \gg \lambda_{p}$, and $H \ll \lambda_{T}$.

The importance of stochastic surface roughness can be deduced from our calculations. The relative corrections $\mathcal{E}_{\text {cf }} / \mathcal{E}$ to the Casimir energy due to roughness of amplitude $a$ and transverse correlation length $\xi$ should be of the form (to second order in $a$ ) $a^{2} / H^{2}$ for $\xi \gg H$, and $a^{2} /(\xi H)$ for $\xi \ll H$. The latter behavior is in accordance with Ref. [18]. The experimental case corresponds to neither extreme, making a more complete analysis necessary.

Given the above mentioned limitations, as well as the technical difficulties in achieving the desirable geometries in experiments, it seems that it is difficult to conclusively establish the nontrivial boundary dependence of the Casimir force. Figure 3 shows that in the experiment of Roy and Mohideen [49], the lengths $a$ and $\lambda$ are such that the nontrivial dependence of the Casimir force on the boundary shape is rather weak within the monitored range of $H / a$, and a pairwise summation of two body forces is a possibly adequate approximation. Our results suggest that a set-up with $\lambda$ of the order of $a$ is better suited for observing the nontrivial geometry dependence predicted above.

In general, one expects that as long as the nontrivial features in the geometry of the plates appear only as small perturbations to the trivial flat-plate geometry, the corresponding many-body effects of these features will be hard to measure. Considerably larger effects could result, however, in patterned surfaces with geometrical features that come close together across various parts of the surfaces. In such circumstances a non-perturbative calculation of the forces becomes necessary. Indeed it is most desirable to find robust numerical schemes (possibly along the lines of Ref. [57]) that can also incorporate the finite conductivity effects and surface roughness typical for experimental set-ups.

Finally, we note that in the set-up of Fig. 1, nontrivial shape dependencies appear as corrections to a larger Casimir force. For the purpose of experimental tests, it is much more desirable to devise set-ups which directly probe differences, without the need for subtracting a larger baseline force. For example, in an atomic force experiment, simultaneous scanning of a flat and corrugated substrate would be desirable; while in the torsion pendulum experiment, one can imagine suspending a spherical lens equidistantly from two plates, one of which is corrugated.

\section{Acknowledgments}

We thank J.-P. Bouchaud, S. Dietrich, S. G. Johnson, U. Mohideen, M. L. Povinelli, and S. Scheidl for useful discussions. This work was supported by the Deutsche Forschungsgemeinschaft through the Emmy Noether grant No. EM70/2-1 (T.E.), through grant No. HA3030/1-2 (A.H., at MIT), and by the National Science Foundation through grant No. DMR-01-18213 (T.E. and M.K.). 


\section{APPENDIX A: PATH INTEGRAL FORMULATION FOR PARTITION FUNCTIONS}

We consider $N$ manifolds (objects) $\Omega_{\alpha}$ with $\alpha=1, \ldots, N$. Each point on the manifold $\Omega_{\alpha}$ is represented by a vector $X_{\alpha}(\mathbf{y})=\left(X_{\alpha}^{\mu}(\mathbf{y}) ; \mu=1, \ldots, d\right)$; a $D$ dimensional manifold $\Omega_{\alpha}$ embedded in $d$ dimensional space is parameterized by $\mathbf{y}=\left(y_{1}, \ldots, y_{D}\right)$.

\section{Dirichlet boundary conditions}

The Dirichlet boundary condition $\Phi=0$ on the manifolds, can be enforced by the functional $\prod_{X_{\alpha}} \delta\left[\Phi\left(X_{\alpha}\right)\right]$ in Eq. 6a), which can be expressed in terms of auxiliary fields $\Psi_{\alpha}\left(X_{\alpha}\right)$ as 47, 55]

$$
\prod_{X_{\alpha}} \delta\left[\Phi\left(X_{\alpha}\right)\right] \equiv \int \mathcal{D} \Psi_{\alpha}\left(X_{\alpha}\right) \exp \left[i \int_{\Omega_{\alpha}} d X_{\alpha} \Psi_{\alpha}\left(X_{\alpha}\right) \Phi\left(X_{\alpha}\right)\right] .
$$

The Gaussian integration over $\Phi$ in Eq. (6a) can then be performed, resulting in

$$
\mathcal{Z}_{\mathrm{D}}=\int \prod_{\alpha=1}^{N} \mathcal{D} \Psi_{\alpha}\left(X_{\alpha}\right) e^{-\widetilde{S}_{\text {eff }}\{\Psi\}} .
$$

The effective action $\widetilde{S}_{\text {eff }}$ is given by

$$
\widetilde{S}_{\mathrm{eff}}\{\Psi\}=\frac{1}{2} \sum_{\alpha \beta} \int_{\Omega_{\alpha}} d X_{\alpha} \int_{\Omega_{\beta}} d X_{\beta} \Psi_{\alpha}\left(X_{\alpha}\right) G\left(X_{\alpha}, X_{\beta}\right) \Psi_{\beta}\left(X_{\beta}\right),
$$

where $G\left(\underline{r}, \underline{r}^{\prime}\right)$ is the two-point correlation function in unbounded bulk space. The functional integration over the curved manifolds $\Omega_{\alpha}$ in Eq. (A2) is facilitated by introducing the new fields $\psi_{\alpha}(\mathbf{y}) \equiv \Psi_{\alpha}\left[X_{\alpha}(\mathbf{y})\right]$. However, this transformation requires some care regarding the integration measure $\int_{\Omega_{\alpha}} d X_{\alpha}$ in Eq. (A3), as well as the functional measure $\int \mathcal{D} \Psi_{\alpha}\left(X_{\alpha}\right)$ in Eq. A22). The result is [56, 64.

$$
\int \prod_{\alpha} \mathcal{D} \Psi_{\alpha}\left(X_{\alpha}\right) e^{-\widetilde{S}_{\mathrm{eff}}\{\Psi\}}=\int \prod_{\alpha} \mathcal{D} \phi_{\alpha}(\mathbf{y}) e^{-S_{\mathrm{eff}}\{\phi\}},
$$

where the field $\phi_{\alpha}(\mathbf{y}) \equiv\left[g_{\alpha}(\mathbf{y})\right]^{1 / 4} \psi_{\alpha}(\mathbf{y})$ is given for each manifold $\Omega_{\alpha}$ in terms of the determinant $g_{\alpha}(\mathbf{y})$ of its induced metric

$$
g_{\alpha, i j}(\mathbf{y})=\sum_{\mu=1}^{4} \frac{\partial X_{\alpha}^{\mu}}{\partial y_{i}} \frac{\partial X_{\alpha}^{\mu}}{\partial y_{j}} .
$$

The new effective action $S_{\text {eff }}$ is then given by

$$
S_{\mathrm{eff}}\{\phi\}=\frac{1}{2} \sum_{\alpha \beta} \int d^{D} y \int d^{D} y^{\prime} \phi_{\alpha}(\mathbf{y}) \Gamma_{\alpha \beta}\left(\mathbf{y}, \mathbf{y}^{\prime}\right) \phi_{\beta}\left(\mathbf{y}^{\prime}\right),
$$

where

$$
\Gamma_{\alpha \beta}\left(\mathbf{y}, \mathbf{y}^{\prime}\right)=\left[g_{\alpha}(\mathbf{y})\right]^{1 / 4} G\left[X_{\alpha}(\mathbf{y}), X_{\beta}\left(\mathbf{y}^{\prime}\right)\right]\left[g_{\beta}\left(\mathbf{y}^{\prime}\right)\right]^{1 / 4} .
$$

The functional measure $\int \mathcal{D} \phi_{\alpha}(\mathbf{y})$ on the right hand side of Eq. (A4) is the one conventionally used on a flat manifold (the local coordinate system). The corresponding Gaussian integrations can thus be performed, resulting in Eqs. (7) and (8a), with $\Gamma_{\mathrm{D}} \equiv \Gamma$ from Eq. (A7). Note that in the present formulation, the trace and products of $\Gamma$ are carried out by integrating $\mathbf{y}$ over the flat manifold of the local coordinate system. Any dependence of $\mathcal{Z}_{\mathrm{D}}$ on the metric $g_{\alpha, i j}(\mathbf{y})$ is contained explicitly in the definition of $\Gamma$ in Eq. (A7). 


\section{Neumann boundary conditions}

For the Neumann boundary condition $\partial_{n} \Phi=0$ on the manifolds, the boundary condition enforcing functional $\prod_{X_{\alpha}} \delta\left[\partial_{n} \Phi\left(X_{\alpha}\right)\right]$ in Eq. (6b) can again be expressed in terms of the auxiliary fields $\Psi_{\alpha}\left(X_{\alpha}\right)$ as

$$
\begin{aligned}
\prod_{X_{\alpha}} \delta\left[\partial_{n} \Phi\left(X_{\alpha}\right)\right] & \equiv \int \mathcal{D} \Psi_{\alpha}\left(X_{\alpha}\right) \exp \left[i \int_{\Omega_{\alpha}} d X_{\alpha} \Psi_{\alpha}\left(X_{\alpha}\right) \partial_{n} \Phi\left(X_{\alpha}\right)\right] \\
& =\int \mathcal{D} \Psi_{\alpha}\left(X_{\alpha}\right) \exp \left[-i \int_{\Omega_{\alpha}} d X_{\alpha}\left[\partial_{n} \Psi_{\alpha}\left(X_{\alpha}\right)\right] \Phi\left(X_{\alpha}\right)\right]
\end{aligned}
$$

where the second line follows from an integration by parts. The Gaussian integration over $\Phi$ in Eq. (6b) can then be performed, resulting in

$$
\mathcal{Z}_{\mathrm{N}}=\int \prod_{\alpha=1}^{N} \mathcal{D} \Psi_{\alpha}\left(X_{\alpha}\right) e^{-\widetilde{S}_{\text {eff }}\{\Psi\}}
$$

with the effective action

$$
\begin{aligned}
\widetilde{S}_{\mathrm{eff}}\{\Psi\} & =\frac{1}{2} \sum_{\alpha \beta} \int_{\Omega_{\alpha}} d X_{\alpha} \int_{\Omega_{\beta}} d X_{\beta}\left[\partial_{n} \Psi_{\alpha}\left(X_{\alpha}\right)\right] G\left(X_{\alpha}, X_{\beta}\right)\left[\partial_{n} \Psi_{\beta}\left(X_{\beta}\right)\right] \\
& =\frac{1}{2} \sum_{\alpha \beta} \int_{\Omega_{\alpha}} d X_{\alpha} \int_{\Omega_{\beta}} d X_{\beta} \Psi_{\alpha}\left(X_{\alpha}\right)\left[\partial_{n_{\alpha}} \partial_{n_{\beta}} G\left(X_{\alpha}, X_{\beta}\right)\right] \Psi_{\beta}\left(X_{\beta}\right) .
\end{aligned}
$$

Calculations along similar lines as in the previous paragraph then lead to Eqs. (7) and (8b).

\section{APPENDIX B: CALCULATION OF THE KERNELS}

\section{Dirichlet boundary conditions}

The kernels for Dirichlet boundary conditions were defined in Eqs. (14) in terms of the functions in Eqs. (15). The explicit form of these functions is given by

$$
\begin{aligned}
& F_{1}(y)=-\frac{1}{\pi^{2} y^{4}}-\frac{\pi}{8 H^{3} y} \frac{\cosh (s)}{\sinh ^{3}(s)} \\
& F_{2}(y)=-\frac{\pi}{16 H^{3} y} \frac{\sinh (s)}{\cosh ^{3}(s)} \\
& F_{3}(y)=2 \partial_{z}^{2} G(y, 0)-\frac{1}{2} F_{1}(y) \\
& F_{4}(y)=-2 F_{2}(y) \\
& F_{5}(y)=-\frac{1}{2} F_{3}(y), \\
& F_{6}(y)=-\partial_{z}^{2} G(y, H)-\frac{1}{2} F_{2}(y)
\end{aligned}
$$

with $s=\pi y /(2 H)$. 


\section{Neumann boundary conditions}

For Neumann boundary conditions the functions appearing in the kernels in Eqs. (20) and defined in Eqs. (22) have the explicit forms

$$
\begin{aligned}
& \mathcal{F}_{1}(y)=2 G(y, 0)+\frac{1}{4 \pi H y} \frac{\cosh (s)}{\sinh (s)}, \\
& \mathcal{F}_{2}(y)=-\frac{\pi}{16 H^{3} y} \frac{\sinh (s)}{\cosh ^{3}(s)}, \\
& \mathcal{F}_{3}(y)=g(y, 0)+\frac{\pi}{16 H^{3} y} \frac{\cosh (s)}{\sinh ^{3}(s)}, \\
& \mathcal{F}_{4}(y)=\frac{1}{4 \pi H y} \frac{\sinh (s)}{\cosh (s)}, \\
& \mathcal{F}_{5}(y)=-\frac{1}{2} \partial_{z}^{2} g(y, 0)+\frac{\pi^{3}}{32 H^{5} y} \frac{\cosh ^{3}(s)}{\sinh ^{5}(s)}+\frac{\pi^{3}}{16 H^{5} y} \frac{\cosh (s)}{\sinh ^{5}(s)}, \\
& \mathcal{F}_{6}(y)=-\partial_{z}^{2} g(y, H)-\frac{\pi^{3}}{32 H^{5} y} \frac{\sinh ^{3}(s)}{\cosh ^{5}(s)}+\frac{\pi^{3}}{16 H^{5} y} \frac{\sinh (s)}{\cosh ^{5}(s)}, \\
& \mathcal{F}_{7}(y)=-G(y, 0)+\frac{1}{4} \mathcal{F}_{1}(y), \\
& \mathcal{F}_{8}(y)=-G(y, H)+\frac{1}{4} \mathcal{F}_{4}(y), \\
& \mathcal{F}_{9}(y)=-2 \mathcal{F}_{7}(y) \\
& \mathcal{F}_{10}(y)=-\frac{1}{2} \mathcal{F}_{4}(y) \\
& \mathcal{F}_{11}(y)=-\frac{1}{2} \mathcal{F}_{3}(y), \\
& \mathcal{F}_{12}(y)=-g(y, H)-\frac{1}{2} \mathcal{F}_{2}(y),
\end{aligned}
$$

with $g(y, z)=\partial_{z}^{2} G(y, z)$.

[1] H. B. G. Casimir, Proc. K. Ned. Akad. Wet. 51, 793 (1948).

[2] J. N. Israelachvili, Intermolecular and Surface Forces (Academic, London, 1992).

[3] K. A. Milton, Phys. Rev. D 22, 1441; 22, 1444 (1980).

[4] A. A. Bytsenko, G. Cognola, L. Vanzo, S. Zerbini, Phys. Rep. 266, 1 (1996).

[5] G. Plunien, B. Müller, and W. Greiner, Phys. Rep. 134, 87 (1986).

[6] E. Elizalde and A. Romeo, Am. J. Phys. 59, 711 (1991).

[7] P. W. Milonni, The Quantum Vacuum (Academic, San Diego, 1994).

[8] V. M. Mostepanenko and N. N. Trunov, The Casimir Effect and its Applications (Clarendon, Oxford, 1997).

[9] S. K. Lamoreaux, Am. J. Phys. 67, 850 (1999) (resource letter). 
[10] M. Kardar and R. Golestanian, Rev. Mod. Phys. 71, 1233 (1999).

[11] M. Bordag, U. Mohideen, V. M. Mostepanenko, Phys. Rep. 353, 1 (2001).

[12] M. J. Sparnaay, Physica (Utrecht) 24, 751 (1958).

[13] P. H. G. M. Van Blokland and J. T. G. Overbeek, J. Chem. Soc. Faraday Trans. I 74, 2637 (1978).

[14] S. K. Lamoreaux, Phys. Rev. Lett. 78, 5 (1997); 81, 5475(E) (1998).

[15] U. Mohideen and A. Roy, Phys. Rev. Lett. 81, 4549 (1998); A. Roy and U. Mohideen, Phys. Rev. Lett. 82, 4380 (1999); A. Roy, C.-Y. Lin, and U. Mohideen, Phys. Rev. D 60, 111101 (1999); B.W. Harris, F. Chen, and U. Mohideen, Phys. Rev. A 62, 052109 (2000).

[16] H. B. Chan, V. A. Aksyuk, R. N. Kleiman, D. J. Bishop, and F. Capasso, Science 291, 1941 (2001).

[17] S. K. Karepanov, M. Y. Novikov, and A. S. Sorin, Nuovo Cimento B 100, 411 (1987).

[18] M. Y. Novikov, A. S. Sorin, and V. Y. Chernyak, Theor. Math. Phys. 82, 124 (1990); 82, 252 (1990); 91, 658 (1992); 92, 773 (1992).

[19] S. K. Lamoreaux, Phys. Rev. A 59, R3149 (1999).

[20] A. Lambrecht and S. Reynaud, Eur. Phys. J. D 8, 309 (2000).

[21] M. Bordag, B. Geyer, G. L. Klimchitskaya, and V. M. Mostepanenko, Phys. Rev. Lett. 85, 503 (2000); V. B. Bezerra, G. L. Klimchitskaya, and V. M. Mostepanenko, Phys. Rev. A 65, 052113 (2002); B. Geyer, G. L. Klimchitskaya, and V. M. Mostepanenko, Phys. Rev. A 65, 062109 (2002).

[22] B. Derjaguin, Kolloid Z. 69, 155 (1934).

[23] G. Bressi, G. Carugno, R. Onofrio, and G. Ruoso, Phys. Rev. Lett. 88, 041804 (2002).

[24] M. E. Fisher and P. G. de Gennes, C. R. Acad. Sc. Paris B 287, 207 (1978); P. G. de Gennes, C. R. Acad. Sci. Ser. II 292, 701 (1981).

[25] D. Beysens and D. Estève, Phys. Rev. Lett. 54, 2123 (1985); for a review see D. Beysens, J.-M. Petit, T. Narayanan, A. Kumar, and M. L. Broide, Ber. Bunsenges. Phys. Chem. 98, 382 (1994).

[26] P. Ziherl, R. Podgornik, and S. Žumer, Phys. Rev. Lett. 82, 1189 (1999); A. Borštnik, H. Stark, and S. Žumer, Phys. Rev. E 61, 2831 (2000); R. Golestanian, A. Ajdari, and J.-B. Fournier, Phys. Rev. E 64, 022701 (2001).

[27] N. Uchida, Phys. Rev. Lett. 87, 216101 (2001).

[28] R. Golestanian, M. Goulian, and M. Kardar, Europhys. Lett. 33, 241 (1996); R. Golestanian, Europhys. Lett. 36, 1217 (1996); R. R. Netz, J. Phys. I 7, 833 (1997); P. G. Dommersnes and J.-B. Fournier, Europhys. Lett. 46, 256 (1999); W. Helfrich and T.R. Weikl, Eur. Phys. J. E 5, 423 (2001).

[29] M. Krech and S. Dietrich, Phys. Rev. Lett. 66, 245 (1991); 67, 1055 (1991); Phys. Rev. A 46, 1922 (1992); 46, 1886 (1992); J. Low Temp. Phys. 89, 145 (1992).

[30] M. Krech, The Casimir Effect in Critical Systems (World Scientific, Singapore, 1994); J. Phys.: Condens. Matter 11, R391 (1999).

[31] R. Golestanian, Phys. Rev. E 62, 5242 (2000).

[32] A. Hanke, F. Schlesener, E. Eisenriegler, and S. Dietrich, Phys. Rev. Lett. 81, 1885 (1998); F. Schlesener, A. Hanke, and S. Dietrich, cond-mat/0202532.

[33] D. Bartolo, A. Ajdari, J.-B. Fournier, and R. Golestanian, cond-mat/0208095, accepted for publication in Phys. Rev. Lett.

[34] A. Mukhopadhyay and B. M. Law, Phys. Rev. Lett. 83, 772 (1999).

[35] R. Garcia and M. H. W. Chan, Phys. Rev. Lett. 83, 1187 (1999); Physica B 280, 55 (2000); J. Low Temp. Phys. 121, 495 (2000); Phys. Rev. Lett. 88, 086101 (2002).

[36] E. M. Lifshitz, Sov. Phys. JETP 2, 73 (1956); I. E. Dzyaloshinskii, E. M. Lifshitz, and L. P. Pitaevskii, Adv. Phys. 10, 165 (1961).

[37] However, one should note that by virtue of Schwinger's source theory [J. Schwinger, L. L. DeRaad, Jr., and K. A. Milton, Ann. Phys. (New York) 115, 1 (1978)], the Casimir force can be obtained without referring to vacuum fluctuations; see also Ref. [0].

[38] T. H. Boyer, Phys. Rev. 174, 1764 (1968).

[39] R. Balian and B. Duplantier, Ann. Phys. (New York) 104, 300 (1977); 112, 165 (1978).

[40] G. J. Maclay, Phys. Rev. A 61, 052110 (2000).

[41] However, for a different interpretation of the result for Casimir energies, see N. Graham, R. L. Jaffe, V. Khemani, M. Quandt, M. Scandurra, and H. Weigel, preprint hep-th/0207205.

[42] T. H. Boyer, Phys. Rev. A 9, 2078 (1974); V. Hushwater, Am. J. Phys. 65, 381 (1997); O. Kenneth, I. Klich, A. Mann, and M. Revzen, Phys. Rev. Lett. 89, 033001 (2002).

[43] Y. Srivastava, A. Widom, and M. H. Friedman, Phys. Rev. Lett. 55, 2246 (1985); see also the Comment by M. A. Stroscio, Phys. Rev. Lett. 56, 2107 (1986).

[44] F. M. Serry, D. Walliser, and G. J. Maclay, J. Microelectromech. Syst. 4, 193 (1995); J. Appl. Phys. 84, 2501 (1998).

[45] E. Buks and M. L. Roukes, Phys. Rev. B 63, 033402 (2001); Nature 419, 119 (2002).

[46] H. B. Chan, V. A. Aksyuk, R. N. Kleiman, D. J. Bishop, and F. Capasso, Phys. Rev. Lett. 87, 211801 (2001).

[47] R. Golestanian and M. Kardar, Phys. Rev. Lett. 78, 3421 (1997); Phys. Rev. A 58, 1713 (1998).

[48] T. Emig, A. Hanke, R. Golestanian, and M. Kardar, Phys. Rev. Lett. 87, 260402 (2001).

[49] A. Roy and U. Mohideen, Phys. Rev. Lett. 82, 4380 (1999).

[50] This configuration has also been considered to study geometrically-controlled twist transitions in cells of nematic liquid crystals, see P. Patricio, M. M. T. da Gama, and S. Dietrich, Phys. Rev. Lett. 88, 245502 (2002).

[51] G. L. Klimchitskaya, S. I. Zanette, and A. O. Caride, Phys. Rev. A 63, 14101 (2000).

[52] F. Chen, U. Mohideen, G. L. Klimchitskaya, and V. M. Mostepanenko, Phys. Rev. Lett. 88, 101801 (2002); Phys. Rev. A 
66, 032113 (2002).

[53] See, e.g., M. E. Peskin and D. V. Schroeder, An Introduction to Quantum Field Theory (Addison-Wesley, Reading, 1995), Chapter 9.

[54] J. D. Jackson, Classical Electrodynamics (Wiley, New York, 1999).

[55] H. Li and M. Kardar, Phys. Rev. Lett. 67, 3275 (1991); Phys. Rev. A 46, 6490 (1992).

[56] A. Hanke and M. Kardar, Phys. Rev. Lett. 86, 4596 (2001); Phys. Rev. E 65, 046121 (2002).

[57] T. Emig, preprint cond-mat/0206585.

[58] M. Bordag, G. L. Klimchitskaya, and V. M. Mostepanenko, Mod. Phys. Lett. A 9, 2515 (1994); Int. J. Mod. Phys. A 10, 2661 (1995).

[59] The results for the Dirichlet kernels $K$ and $Q$ quoted in Ref. 47] deviate from Eqs. (14a) and (14b) since the last terms in each of the two lines of Eq. (15) in the second reference 47 are interchanged.

[60] J. Schwinger, L. L. DeRaad, Jr., and K. A. Milton, Ann. Phys. (New York) 115, 1 (1978).

[61] G. Arfken, Mathematical Methods for Physicists, Sec. 7.2 (Academic, New York, 1970).

[62] A. Erdelyi, W. Magnus, F. Oberhettinger, and F. G. Tricomi, Higher Transcendental Functions, Vol. 1 (Krieger, New York, 1981).

[63] S. Laporta and E. Remiddi, Phys. Lett. B 379, 283 (1996).

[64] See, e.g., F. David in Statistical Mechanics of Membranes and Surfaces, edited by D. Nelson, T. Piran, and S. Weinberg (World Scientific, Singapore, 1989). 